\title{
Docetaxel plus S-1 versus cisplatin plus S-1 in unresectable gastric cancer without measurable lesions: a randomized phase II trial (HERBIS-3)
}

\author{
Yukinori Kurokawa ${ }^{1} \cdot$ Jin Matsuyama ${ }^{2} \cdot$ Kazuhiro Nishikawa $^{3} \cdot$ Atsushi Takeno $^{4} \cdot$ Yutaka Kimura $^{5}$. \\ Kazumasa Fujitani $^{6} \cdot$ Ryohei Kawabata $^{7} \cdot$ Yoichi Makari $^{8} \cdot$ Tetsuji Terazawa $^{9} \cdot$ Hisato Kawakami $^{10}$. Daisuke Sakai ${ }^{11}$. \\ Toshio Shimokawa ${ }^{12} \cdot$ Taroh Satoh $^{11}$
}

Received: 16 June 2020 / Accepted: 1 August 2020 / Published online: 10 August 2020

(c) The International Gastric Cancer Association and The Japanese Gastric Cancer Association 2020

\begin{abstract}
Background Cisplatin plus S-1 (CS) is the standard first-line chemotherapy for advanced gastric cancer (AGC) in Japan. A previous phase III trial showed that docetaxel plus S-1 (DS) was effective for AGC without measurable lesions, but no studies have compared these two regimens.

Methods Eligible patients had unresectable or recurrent HER2-negative AGC without measurable lesions. Patients were randomized to DS (docetaxel $40 \mathrm{mg} / \mathrm{m}^{2}$ on day $1, \mathrm{~S}-180-120 \mathrm{mg}$ on days $1-14$, every 3 weeks) or CS (cisplatin $60 \mathrm{mg} / \mathrm{m}^{2}$ on day $8, \mathrm{~S}-1$ 80-120 $\mathrm{mg}$ on days 1-21, every 5 weeks). The primary endpoint was overall survival (OS).

Results All patients had unresectable primary disease. Sixty-one patients were randomly assigned to DS $(n=30)$ or CS $(n=31)$. One CS patient was ineligible due to HER2 positivity. The median number of cycles was 9.5 (range 2-49) with DS and 5.5 (range 1-10) with CS. There were no treatment-related deaths. The most common grade 3-4 non-hematological toxicity was fatigue (7\% with DS, $13 \%$ with CS), followed by anorexia (3\% with DS, $10 \%$ with CS) and diarrhea ( $3 \%$ with DS, $10 \%$ with CS). The 2-year OS rates were $43.3 \%$ with DS and $30.0 \%$ with CS (log-rank $P=0.113$ ), with a hazard ratio of 0.617 (95\% confidence interval $0.337-1.128)$, indicating non-inferiority of DS to CS with respect to OS $(P<0.001)$.

Conclusions DS showed slightly but nonsignificantly less toxicity and higher efficacy than CS for AGC without measurable lesions. DS should be further investigated in phase III trials.
\end{abstract}

Keywords S-1 $\cdot$ Cisplatin $\cdot$ Docetaxel $\cdot$ R1 gastrectomy $\cdot$ HERBIS-3

Yukinori Kurokawa

ykurokawa@gesurg.med.osaka-u.ac.jp

1 Department of Gastroenterological Surgery, Osaka University Graduate School of Medicine, 2-2-E2,

Yamadaoka, Suita, Osaka 565-0871, Japan

2 Department of Surgery, Yao Municipal Hospital, Osaka, Japan

3 Department of Surgery, National Hospital Organization Osaka National Hospital, Osaka, Japan

4 Department of Surgery, Kansai Rosai Hospital, Hyogo, Japan

5 Department of Surgery, Sakai City Medical Center, Osaka, Japan

6 Department of Surgery, Osaka General Medical Center, Osaka, Japan
7 Department of Surgery, Osaka Rosai Hospital, Osaka, Japan

8 Department of Surgery, Minoh City Hospital, Osaka, Japan

9 Cancer Chemotherapy Center, Osaka Medical College, Osaka, Japan

10 Department of Medical Oncology, Faculty of Medicine, Kindai University, Osaka, Japan

11 Department of Frontier Science for Cancer and Chemotherapy, Osaka University Graduate School of Medicine, Osaka, Japan

12 Clinical Study Support Center, Wakayama Medical University, Wakayama, Japan 


\section{Introduction}

Gastric cancer is the second leading cause of cancer deaths worldwide [1]. Although surgical resection is the only curative treatment, many patients suffer from unresectable or recurrent disease [2-4]. Since the prognoses of patients with advanced gastric cancer (AGC) are still poor, more effective chemotherapy regimens should be developed. At present, a fluoropyrimidine plus a platinum compound is the most widely used type of first-line regimen for human epidermal growth factor receptor type 2 (HER2)-negative AGC, in both the West and the East [5-7].

$\mathrm{S}-1$ is a fluoropyrimidine compound that contains tegafur, gimeracil, and oteracil potassium and inhibits dihydropyrimidine dehydrogenase [8]. A phase III (JCOG9912) trial demonstrated that S-1 alone was not inferior to fluorouracil alone for AGC [9]. Another phase III (SPIRITS) trial demonstrated that the cisplatin plus S-1 (CS) regimen was significantly superior to $\mathrm{S}-1$ alone for AGC, which led to its establishment as the standard first-line therapy in Japan [6]. On the other hand, followup analysis of an international phase III (START) trial revealed that the docetaxel and $\mathrm{S}-1$ (DS) regimen significantly improved survival compared with $\mathrm{S}-1$ alone, although the initial analysis failed to show a significant difference in overall survival (OS) [10]. That study also showed that the DS regimen had relatively mild toxicity, and importantly, its efficacy was much higher for patients without measurable lesions compared to those with them. If the DS regimen has similar efficacy to the standard CS regimen in this subgroup of AGC patients, it could replace $\mathrm{CS}$ as the standard first-line regimen due to its relatively low toxicity. Here, we describe a randomized phase II trial to compare the efficacy and safety of these two regimens for AGC without measurable lesions.

\section{Methods}

\section{Patients}

This study was a randomized, open-label, phase II trial conducted at 16 institutions in the Osaka Gastrointestinal Cancer Chemotherapy Study Group (OGSG). We recruited patients with histologically proven unresectable or recurrent gastric cancer without measurable lesions as defined by the Response Evaluation Criteria in Solid Tumors (RECIST), version 1.1 [11]. Patients who underwent R1 or $\mathrm{R} 2$ resection within 6 weeks before registration were also eligible. Eligibility criteria included the following: (1) age between 20 and 75 years; (2) Eastern Cooperative
Oncology Group (ECOG) performance status (PS) score of 0-2; (3) HER2 negativity or HER2 status unknown; (4) life expectancy longer than 3 months; and (5) adequate organ function, including leukocyte count between 3,000 and $12,000 / \mathrm{mm}^{3}$, neutrophil count $\geq 2,000 / \mathrm{mm}^{3}$, platelet count $\geq 100,000 / \mathrm{mm}^{3}$, hemoglobin $\geq 9.0 \mathrm{~g} / \mathrm{dL}$, serum aspartate aminotransferase (AST) and alanine aminotransferase (ALT) both $<100$ IU/L, serum total bilirubin $\leq 1.5 \mathrm{mg} / \mathrm{dL}$, serum creatinine $\leq 1.2 \mathrm{mg} / \mathrm{dL}$, and creatinine clearance $(\mathrm{CCr}) \geq 60 \mathrm{~mL} / \mathrm{min}$. Patients were excluded from the trial if they had inadequate oral intake; demonstrated massive peritoneal metastasis, brain metastasis, or active bleeding from main tumor; or had received prior chemotherapy or radiotherapy. All patients provided written informed consent before enrollment. The study protocol was approved by the Osaka Gastrointestinal Cancer Chemotherapy Study Group (OGSG) Steering Committee and the institutional review boards of all participating hospitals. This study was registered with UMIN-CTR, UMIN000006179.

\section{Treatment}

Patients were randomly assigned (1:1) to receive either DS or CS using the minimization method, with stratification by institution and whether the malignancy was unresectable versus recurrent. The allocated treatments were not masked from the investigators or patients.

In the DS group, patients received docetaxel (40 mg/ $\mathrm{m}^{2}$ ) intravenously on day 1 and oral S-1 twice daily at a dose based on body surface area $\left(<1.25 \mathrm{~m}^{2}, 40 \mathrm{mg} ; \geq 1.25\right.$ to $<1.5 \mathrm{~m}^{2}, 50 \mathrm{mg} ; \geq 1.5 \mathrm{~m}^{2}, 60 \mathrm{mg}$ ) on days $1-14$ of a 3 -week cycle. In the CS group, patients received cisplatin $\left(60 \mathrm{mg} / \mathrm{m}^{2}\right)$ intravenously on day 8 and oral S-1 twice daily at a dose based on body surface area $\left(<1.25 \mathrm{~m}^{2}\right.$, $40 \mathrm{mg} ; \geq 1.25$ to $<1.5 \mathrm{~m}^{2}, 50 \mathrm{mg} ; \geq 1.5 \mathrm{~m}^{2}, 60 \mathrm{mg}$ ) on days 1-21 of a 5-week cycle. These schedules were repeated until disease progression, unacceptable toxicity, or patient withdrawal of consent.

If patients had a neutrophil count $<1000 / \mathrm{mm}^{3}$, platelet count $<50 \times 10^{3} / \mathrm{mm}^{3}$, AST or ALT $>100$ IU/L, total bilirubin $>3.0 \mathrm{mg} / \mathrm{dl}, \mathrm{CCr}<50 \mathrm{ml} / \mathrm{min}$, fever lasting longer than 3 days, grade 2 fatigue, anorexia, diarrhea, nausea, vomiting, oral mucositis or rash, or any adverse events of grade 3 or higher, treatment with S-1 was suspended.

\section{Evaluations}

The primary endpoint was OS. The secondary endpoints were progression-free survival (PFS) and adverse events. Tumors were assessed every 2 months until disease progression. OS was defined as the time from the date of enrollment to the date of death from any cause. PFS was defined as the 
time from the date of enrollment to the date of disease progression, relapse, or death from any cause. Adverse events were evaluated according to the National Cancer Institute Common Terminology Criteria for Adverse Events (NCICTCAE), version 4.0.

\section{Statistical analysis}

This randomized phase II trial using a screening design was designed to evaluate the non-inferiority of DS relative to CS in terms of OS in patients with AGC without measurable lesions. The 2-year OS rate with CS was $23.6 \%$ in the SPIRITS trial [6]. Since the prognosis in AGC is usually better when there are no measurable lesions $[9,10]$, we assumed 2 -year OS rates of $30 \%$ in both treatment groups. An initial sample size of 100 patients was expected to achieve a power of $80 \%$ with a one-sided alpha error of 0.1 and a noninferiority margin (hazard ratio (HR)) of 1.60. The accrual period was 3 years, and the follow-up period was 2 years. After 3 years of slow accrual, the OGSG Steering Committee approved an amendment to reduce the sample size. The amended sample size was 60 , with a one-sided alpha error of 0.2 , and there was a 5-year accrual period with 2-year follow-up.

The primary analysis was based on the full analysis set (FAS), which consists of all randomized patients except those who were found to be ineligible after enrollment. We used the Kaplan-Meier method to estimate survival curves and Greenwood's formula to calculate $95 \%$ confidence intervals (CIs) for survival rates. A log-rank test was applied to comparison of survival curves. A Cox proportional hazards model was applied to calculate HRs and 95\% CIs. A $P$ value $<0.05$ was considered statistically significant. Statistical analyses were conducted with $\mathrm{R}$ version 3.6.1 (the $\mathrm{R}$ Foundation for Statistical Computing, Vienna, Austria) or SAS version 9.4 (SAS Institute, Cary, NC).

\section{Results}

Between September 2011 and September 2015, a total of 61 patients (30 assigned to DS and 31 assigned to CS) were enrolled from 16 hospitals (Fig. 1). One patient did not start protocol treatment because HER2 positivity was revealed after registration. This patient was excluded from the FAS. The characteristics of the 60 patients remaining in the FAS are shown in Table 1. No patients in either group had

Table 1 Patient characteristics

\begin{tabular}{lll}
\hline Characteristic & DS $(n=30)$ & CS $(n=30)$ \\
\hline Age, years & & \\
Median & 64 & 67 \\
Range & $32-75$ & $53-75$ \\
Sex & & \\
Male & $20(67 \%)$ & $18(60 \%)$ \\
Female & $10(33 \%)$ & $12(40 \%)$ \\
Performance status & & $12(40 \%)$ \\
0 & $13(43 \%)$ & $18(60 \%)$ \\
1 & $17(57 \%)$ & $30(100 \%)$ \\
Unresectable/recurrent & & $0(0 \%)$ \\
Unresectable & $30(100 \%)$ & \\
Recurrent & $0(0 \%)$ & $14(47 \%)$ \\
Previous gastrectomy & & $6(20 \%)$ \\
No resection & $15(50 \%)$ & $10(33 \%)$ \\
R1 gastrectomy & $10(33 \%)$ & $21(70 \%)$ \\
R2 gastrectomy & $5(17 \%)$ & $12(40 \%)$ \\
Tumor sites & & $8(27 \%)$ \\
Peritoneum & $19(63 \%)$ & \\
Lymph node & $5(17 \%)$ & $(20 \%)$ \\
Stomach (primary) & $0(0 \%)$ & \\
Bone & & \\
\hline
\end{tabular}

${ }^{\mathrm{a} S}$ Some patients had multiple tumor sites

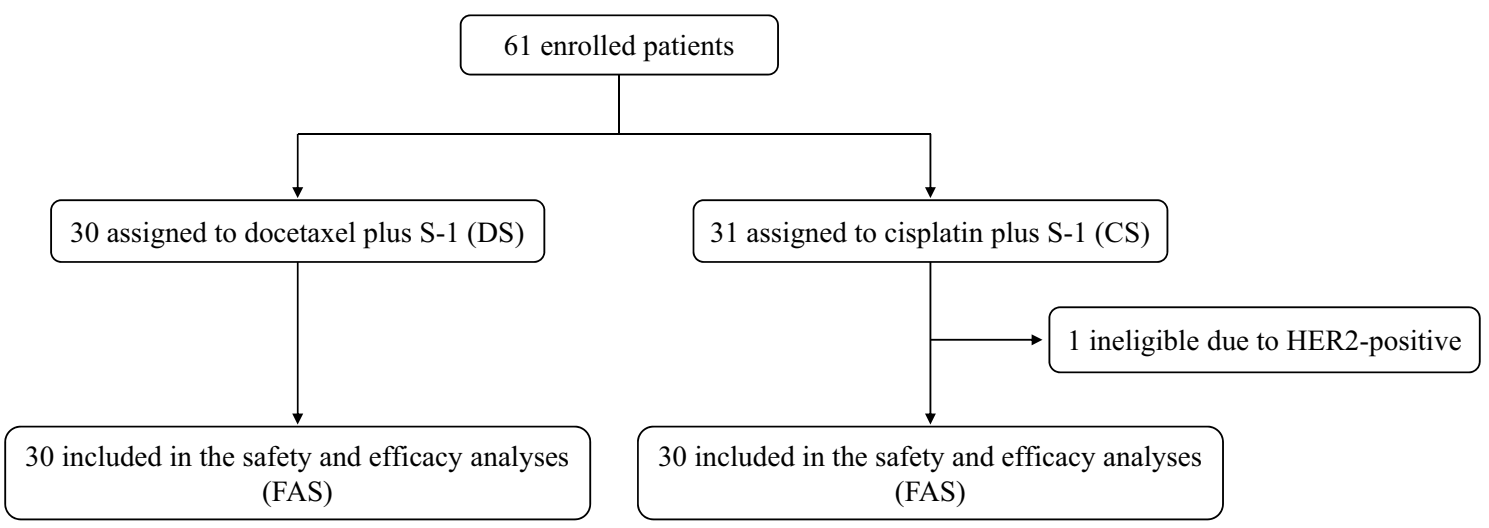

Fig. 1 CONSORT flow diagram for the HERBIS-3 trial 
recurrent tumors. Approximately half of all patients underwent gastrectomy just before enrollment; R1 gastrectomy was performed in ten (33\%) DS patients and six (20\%) CS patients. The most common tumor site in both groups was the peritoneum.

The median number of cycles was 9.5 (range 2-49) with DS and 5.5 (range 1-10) with CS (Table 2).The relative dose intensities of docetaxel and S-1 with DS were $86.1 \%$ and $77.9 \%$, respectively, whereas those of cisplatin and S-1 with CS were $63.3 \%$ and $72.9 \%$, respectively. Major adverse events are shown in Table 3. Among the hematological adverse events, grade 3-4 neutropenia was more common with DS than CS (40\% vs $27 \%$, respectively). On the other hand, non-hematological grade 3-4 toxicities such as fatigue ( $7 \%$ vs $13 \%$, respectively), anorexia (3\% vs $10 \%$, respectively), and diarrhea (3\% vs $10 \%$, respectively) occurred less frequently with DS than with CS. Creatinine was elevated only in the CS group. There were no treatment-related deaths in either group. Subsequent chemotherapy after treatment discontinuation was administered to $26(87 \%)$ patients who

Table 2 Number of treatment cycles and relative dose intensities of the component drugs

\begin{tabular}{lll}
\hline Characteristic & DS $(n=30)$ & CS $(n=30)$ \\
\hline $\begin{array}{l}\text { Number of treatment cycles } \\
\text { Median }\end{array}$ & 9.5 & 5.5 \\
Range & $2-49$ & $1-10$ \\
Relative dose intensities & & \\
S-1 & $77.9 \%$ & $72.9 \%$ \\
Docetaxel & $86.1 \%$ & - \\
Cisplatin & - & $63.3 \%$ \\
\hline
\end{tabular}

received DS and 25 (83\%) patients who received CS. The most common regimens other than S-1 monotherapy were irinotecan alone followed by irinotecan plus cisplatin in the DS group, and nab-paclitaxel followed by paclitaxel plus ramucirumab in the CS group.

The median duration of follow-up for censored cases at the time of analysis was 36.8 months. The median OS durations were 20.0 months (95\% CI 15.3-not estimated) with DS and 15.8 months (95\% CI 11.6-24.2) with CS (logrank $P=0.113$; Fig. 2a). The 2-year OS rates were $43.3 \%$ (28.8-65.2) with DS and 30.0\% (95\% CI 17.4-51.8) with CS, with a HR of 0.617 (95\% CI 0.337-1.128). Non-inferiority of DS to CS with respect to OS was statistically demonstrated $(P<0.001$ for non-inferiority). The median PFS durations were 11.2 months (95\% CI 7.6-20.8) with DS and 9.6 months (95\% CI 7.4-14.0) with CS (log-rank $P=0.196$; Fig. 2b). The 2-year PFS rates were $26.7 \%$ (14.7-48.3) with DS and $13.3 \%$ (95\% CI 5.4-33.2) with CS, with a HR of 0.698 (95\% CI 0.404-1.208).

Subgroup analyses of OS according to age, sex, PS, and previous gastrectomy demonstrated moderate but nonsignificant interaction between treatment effect and previous gastrectomy ( $P=0.059)$ (Fig. 3). In patients who underwent R1 gastrectomy before enrollment, the 2-year OS rates were $70.0 \%$ (95\% CI 46.7-100.0) with DS and $16.7 \%$ (95\% CI 2.8-99.7\%) with CS, with a HR of 0.153 (95\% CI 0.037-0.632) (log-rank $P=0.003$; Fig. 4a); the 2-year PFS rates were 50.0\% (95\% CI 26.9-92.9) with DS and 16.7\% (95\% CI 2.8-99.7\%) with CS, with a HR of 0.216 (95\% CI $0.064-0.730$ ) (log-rank $P=0.008$; Fig. $4 \mathrm{~b}$ ). In 15 of the 16 cases, the R1 status was based on peritoneal lavage cytology findings, while in the remaining case it was due to both cytology results and a positive resection margin.

\begin{tabular}{|c|c|c|c|c|c|c|c|c|c|c|}
\hline & \multicolumn{5}{|c|}{ DS $(n=30)$} & \multicolumn{5}{|c|}{$\mathrm{CS}(n=30)$} \\
\hline & G1 & $\mathrm{G} 2$ & G3 & G4 & $\begin{array}{l}\geq \mathrm{G} 3 \\
(\%)\end{array}$ & G1 & G2 & G3 & G4 & $\begin{array}{l}\geq \mathrm{G} 3 \\
(\%)\end{array}$ \\
\hline Neutropenia & 7 & 3 & 6 & 6 & 40 & 3 & 7 & 7 & 1 & 27 \\
\hline Anemia & 14 & 7 & 2 & 1 & 10 & 9 & 11 & 2 & 1 & 10 \\
\hline AST & 4 & 0 & 1 & 0 & 3 & 3 & 0 & 0 & 0 & 0 \\
\hline ALT & 4 & 0 & 1 & 0 & 3 & 2 & 0 & 1 & 0 & 3 \\
\hline Total bilirubin & 3 & 5 & 1 & 0 & 3 & 1 & 1 & 1 & 0 & 3 \\
\hline Creatinine & 0 & 0 & 0 & 0 & 0 & 7 & 1 & 0 & 0 & 0 \\
\hline Hyponatremia & 1 & 0 & 1 & 0 & 3 & 5 & 0 & 2 & 0 & 7 \\
\hline Febrile neutropenia & 0 & 0 & 2 & 0 & 7 & 0 & 0 & 0 & 0 & 0 \\
\hline Anorexia & 11 & 8 & 1 & 0 & 3 & 11 & 8 & 3 & 0 & 10 \\
\hline Fatigue & 9 & 12 & 2 & 0 & 7 & 8 & 10 & 4 & 0 & 13 \\
\hline Nausea & 9 & 3 & 1 & 0 & 3 & 9 & 5 & 2 & 0 & 7 \\
\hline Diarrhea & 7 & 3 & 1 & 0 & 3 & 3 & 2 & 3 & 0 & 10 \\
\hline Oral mucositis & 6 & 3 & 0 & 0 & 0 & 2 & 2 & 1 & 0 & 3 \\
\hline
\end{tabular}

$A S T$ aspartate aminotransferase, $A L T$ alanine aminotransferase 

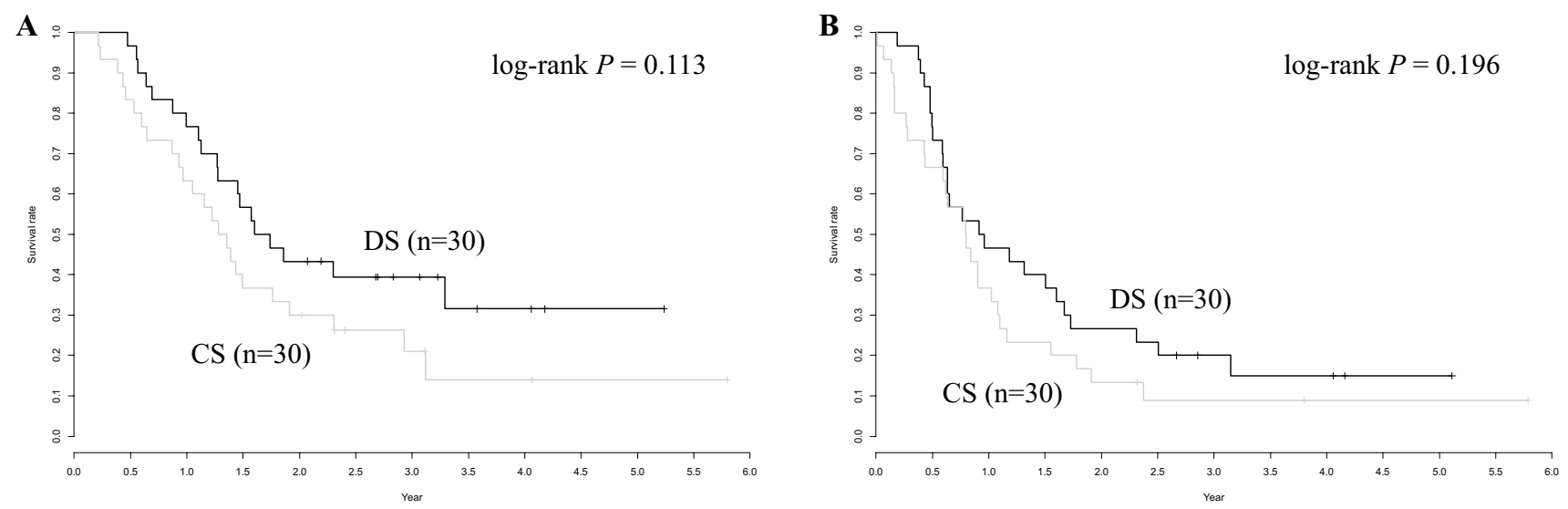

Fig. 2 Kaplan-Meier overall survival (a) and progression-free survival (b) for docetaxel plus S-1 (DS) versus cisplatin plus S-1 (CS)

\begin{tabular}{|c|c|c|c|c|c|c|}
\hline & $\begin{array}{c}\text { DS } \\
(n=30)\end{array}$ & $\begin{array}{c}\text { CS } \\
(n=30)\end{array}$ & & $\operatorname{HR}[95 \% \mathrm{CI}]$ & $P$ & $\begin{array}{c}P \text { for } \\
\text { interaction }\end{array}$ \\
\hline \multicolumn{7}{|l|}{ Age } \\
\hline$<65$ years & 16 & 8 & $\longrightarrow \quad 1$ & $0.76[0.25-2.29]$ & 0.628 & 0.848 \\
\hline$\geq 65$ years & 14 & 22 & $\longrightarrow$ & $0.68[0.31-1.46]$ & 0.314 & \\
\hline \multicolumn{7}{|l|}{ Sex } \\
\hline Male & 20 & 18 & $\longrightarrow$ & $0.70[0.33-1.47]$ & 0.341 & 0.521 \\
\hline Female & 10 & 12 & $\longrightarrow$ & $0.46[0.16-1.36]$ & 0.151 & \\
\hline \multicolumn{7}{|l|}{ Performance status } \\
\hline 0 & 13 & 12 & $\longrightarrow$ & $0.39[0.14-1.09]$ & 0.064 & 0.414 \\
\hline 1 & 17 & 18 & $\longrightarrow$ & $0.76[0.35-1.65]$ & 0.494 & \\
\hline \multicolumn{7}{|l|}{ Previous gastrectomy } \\
\hline No resection & 15 & 14 & \multirow{3}{*}{$\stackrel{0.04}{\longrightarrow}$} & $0.76[0.33-1.73]$ & 0.514 & 0.059 \\
\hline $\mathrm{R} 1$ gastrectomy & 10 & & & $0.17[0.04-0.63]$ & 0.003 & \\
\hline $\mathrm{R} 2$ gastrectomy & 5 & 10 & & $1.18[0.33-4.27]$ & 0.799 & \\
\hline & & & $\stackrel{0.10}{\leftarrow}$ DS better & $\begin{array}{l}.00 \\
\Rightarrow\end{array}$ & & \\
\hline
\end{tabular}

Fig. 3 Forest plot for overall survival in the subgroup analysis. $D S$ docetaxel plus $\mathrm{S}-1, C S$ cisplatin plus S-1

\section{Discussion}

This randomized phase II trial is the first clinical study to compare DS and CS regimens in patients with AGC. Not only was DS associated with superior patient compliance and a favorable toxicity profile compared to CS, but it also showed nonsignificantly better OS and PFS. In patients who underwent R1 gastrectomy before enrollment, both OS and PFS were significantly better with DS than with
CS. The true benefit of DS should be tested in phase III trials.

This study showed that DS resulted in mild toxicity compared with CS. A well-known disadvantage of docetaxel is myelosuppression $[12,13]$. Indeed, grade 3-4 neutropenia occurred more frequently with DS than CS (40\% vs $27 \%$, respectively), but the incidence of febrile neutropenia with DS was only $7 \%$. By contrast, the proportion of patients who suffered from non-hematological toxicities such as fatigue, anorexia, and diarrhea was lower with DS than with CS. 

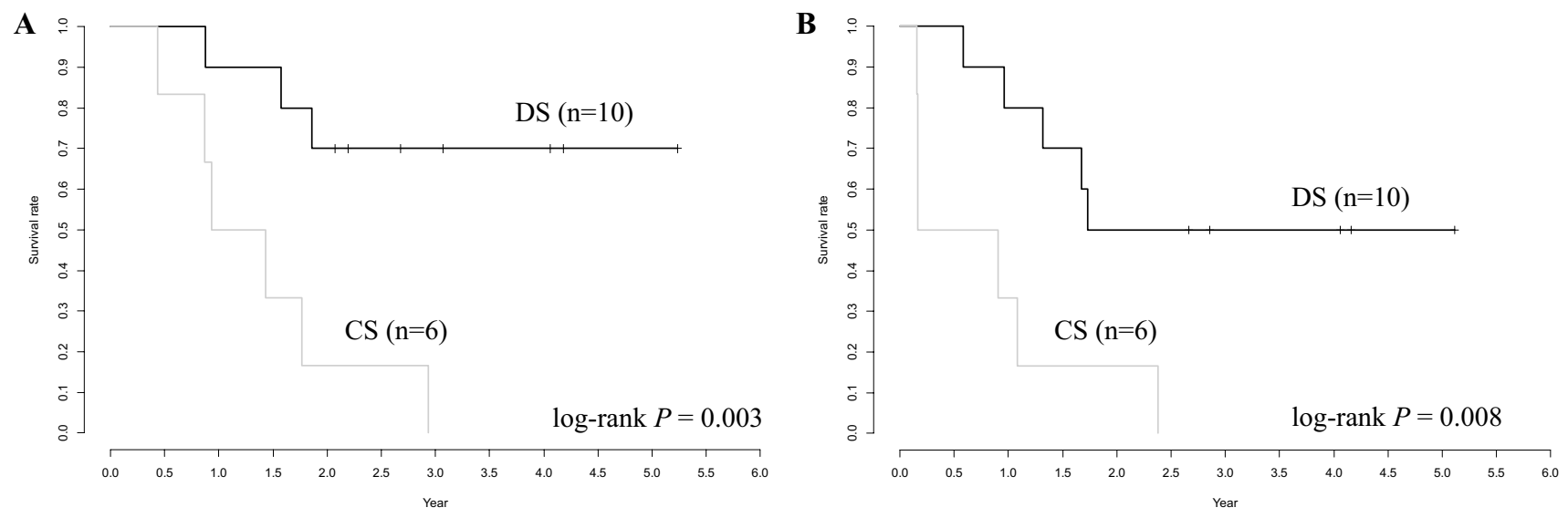

Fig. 4 Kaplan-Meier overall survival (a) and progression-free survival (b) for docetaxel plus S-1 (DS) and cisplatin plus S-1 (CS) in patients who underwent R1 gastrectomy before enrollment

It is suspected that the favorable toxicity profile of DS led to a greater number of treatment cycles and higher relative dose intensities of the component drugs. In addition, DS is more convenient as it can be administered on an outpatient basis. Thus, one possible reason for the favorable prognosis of patients with DS was the better feasibility of this regimen.

Docetaxel is known to be highly effective against peritoneal metastasis because it is transported into the peritoneal cavity $[14,15]$. A phase III START trial that compared DS to $\mathrm{S}-1$ alone for AGC revealed a remarkable interaction between the presence or absence of measurable lesions and treatment effect in a preplanned subgroup analysis [10]. Patients with measurable lesions such as liver or lymph node metastases showed similar OS between DS and S-1 alone, with a HR of 0.904 (95\% CI 0.751-1.088), whereas those with only non-measurable lesions such as peritoneal metastasis showed a significant difference in OS between the two groups, with a HR of 0.649 (95\% CI 0.461-0.914). In addition, our study showed that DS was significantly superior to CS in terms of OS and PFS in patients who underwent R1 gastrectomy due to positive peritoneal lavage cytology, which is a precursor of peritoneal metastasis. Therefore, postoperative chemotherapy using DS might be recommended for patients who have undergone R1 gastrectomy. This strategy is supported by the results of a recent phase III (JACCRO GC-07) trial that demonstrated the significant superiority of DS relative to S-1 alone in terms of relapsefree survival for pathological stage III gastric cancer [16]. Indeed, the 2-year OS rate in patients receiving DS reached $70.0 \%$ in our study, which was much higher than that (46\%) in patients who underwent S-1 monotherapy after R1 gastrectomy in another prospective study [17].

This study had several limitations. First, the statistical power was low due to the small sample size. Nevertheless, the primary endpoint, namely non-inferiority of DS to CS with respect to OS, was statistically proven. However, since this was a small-scale, randomized phase II trial, the noninferiority should be validated in large-scale phase III trials. Second, we initially planned to recruit patients with recurrent AGC, but only enrolled those with unresectable disease. Therefore, the results of this study should not be generalized to recurrent gastric cancer. Third, the DS group included a little more R1 (33\% vs $20 \%$ ) and less R2 (17\% vs 33\%) gastrectomy patients than the CS group. Although the difference between the two groups was not statistically significant, it might affect the survival results.

In conclusion, the results of our randomized phase II trial suggest that DS was less toxic and more effective than CS for unresectable gastric cancer without measurable lesions. Although this study targeted only AGC with no measurable lesions, many patients with unresectable or recurrent AGC have only non-measurable lesions, for instance peritoneal metastasis or positive peritoneal lavage cytology. Our research group, the OGSG, has conducted several prospective studies (the HERBIS series) to establish new treatment strategies for AGC based on both HER2 status and the presence or absence of measurable lesions [18-22]. We expect the clinical value of this strategy to be validated in the future.

Acknowledgements The authors thank all the patients and their family as well as Ms. Akemi Morita, Ms. Mieko Nakai, Ms. Nami Yoshida, and Ms. Chihiro Sawano for data management.

Funding This work was supported by Osaka Clinical Study Supporting Organization.

\section{Compliance with ethical standards}

Conflict of interest Yukinori Kurokawa has received personal fees from Taiho Pharmaceutical, Yakult Honsha, Ono Pharmaceutical, 
MSD, Daiichi Sankyo, Takeda Pharmaceutical, and Kaken Pharmaceutical and research funding from Taiho Pharmaceutical, Ono Pharmaceutical, and MSD. Kazuhiro Nishikawa has received personal fees from Bristol-Myers Squibb, Chugai Pharmaceutical, EA Pharma, Eli Lilly, Ono Pharmaceutical, Taiho Pharmaceutical, and Yakult Honsha. Hisato Kawakami has received personal fees from Bristol-Myers Squibb, Eli Lilly, MSD, Ono Pharmaceutical, Daiichi Sankyo, Taiho Pharmaceutical, AstraZeneca, Bayer, Chugai Pharmaceutical, and Takeda Pharmaceutical and research funding from Chugai Pharmaceutical, Taiho Pharmaceutical, and Eisai. Daisuke Sakai has received personal fees from Chugai Pharmaceutical and Daiichi Sankyo and research funding from Yakult Honsha, Chugai Pharmaceutical, Ono Pharmaceutical, Eli Lilly, Daiichi Sankyo, Astellas, and Incyte. Taroh Satoh has received personal fees from Taiho Pharmaceutical, Ono Pharmaceutical, Chugai Pharmaceutical, Yakult Honsha, Eli Lilly, MSD, Bristol-Myers Squibb, Astellas, Takara-Bio, and Sanofi-Aventis and research funding from Taiho Pharmaceutical, Ono Pharmaceutical, Chugai Pharmaceutical, Yakult Honsha, Eli Lilly, MSD, BristolMyers Squibb, Astellas, Giliad Sciences, Daiichi Sankyo, and Parexell and belongs to endowed department by Ono Pharmaceutical, Chugai Pharmaceutical, and Yakult Honsha. All remaining authors have declared no conflicts of interest.

Ethical approval All procedures performed in studies involving human participants were in accordance with the ethical standards of the institutional research committee and with the 1964 Helsinki declaration and its later amendments. This study was approved by the institutional review boards of all participating hospitals.

Informed consent Written informed consent was provided by all patients before enrollment.

\section{References}

1. Bray F, Ferlay J, Soerjomataram I, et al. Global cancer statistics 2018: GLOBOCAN estimates of incidence and mortality worldwide for 36 cancers in 185 countries. CA Cancer J Clin. 2018;68(6):394-424.

2. Hashimoto T, Kurokawa Y, Mori M, Doki Y. Update on the treatment of gastric cancer. JMA J. 2018;1:40-9.

3. Sasako M, Sano T, Yamamoto S, et al. D2 lymphadenectomy alone or with para-aortic nodal dissection for gastric cancer. $\mathrm{N}$ Engl J Med. 2008;359:453-62.

4. Kurokawa Y, Doki Y, Mizusawa J, et al. Bursectomy versus omentectomy alone for resectable gastric cancer (JCOG1001): a phase 3 , open-label, randomised controlled trial. Lancet Gastroenterol Hepatol. 2018;3:460-8.

5. Cunningham D, Starling N, Rao S, et al. Capecitabine and oxaliplatin for advanced esophagogastric cancer. N Engl J Med. 2008;358:36-46.

6. Koizumi W, Narahara H, Hara T, et al. S-1 plus cisplatin versus $\mathrm{S}-1$ alone for first-line treatment of advanced gastric cancer (SPIRITS trial): a phase III trial. Lancet Oncol. 2008;9:215-21.

7. Kang YK, Kang WK, Shin DB, et al. Capecitabine/cisplatin versus 5-fluorouracil/cisplatin as first-line therapy in patients with advanced gastric cancer: a randomised phase III noninferiority trial. Ann Oncol. 2009;20:666-73.

8. Shirasaka T, Shimamato Y, Ohshimo H, et al. Development of a novel form of an oral 5-fluorouracil derivative (S-1) directed to the potentiation of the tumor selective cytotoxicity of 5-fluorouracil by two biochemical modulators. Anticancer Drugs. 1996;7:548-57.
9. Boku N, Yamamoto S, Fukuda H, et al. Fluorouracil versus combination of irinotecan plus cisplatin versus S-1 in metastatic gastric cancer: a randomised phase 3 study. Lancet Oncol. 2009;10:1063-9.

10. Koizumi W, Kim YH, Fujii M, et al. Addition of docetaxel to S-1 without platinum prolongs survival of patients with advanced gastric cancer: a randomized study (START). J Cancer Res Clin Oncol. 2014;140:319-28.

11. Eisenhauer EA, Therasse P, Bogaerts J, et al. New response evaluation criteria in solid tumours: revised RECIST guideline (version 1.1). Eur J Cancer. 2009;45:228-47.

12. Bang YJ, Kang WK, Kang YK, et al. Docetaxel $75 \mathrm{mg} / \mathrm{m}(2)$ is active and well tolerated in patients with metastatic or recurrent gastric cancer: a phase II trial. Jpn J Clin Oncol. 2002;32:248-54.

13. Mavroudis D, Kourousis C, Androulakis N, et al. Frontline treatment of advanced gastric cancer with docetaxel and granulocyte colony-stimulating factor (G-CSF): a phase II trial. Am J Clin Oncol. 2000;23:341-4.

14. Mai M, Sakata Y, Kanamaru R, et al. A late phase II clinical study of RP56976 (docetaxel) in patients with advanced or recurrent gasyric cancer: a cooperative study group trial (group B). Jpn J Chemother. 1999;26:487-96 (Japanese).

15. Naitoh H, Kawaguchi A, Yamamoto H, et al. Measurement of docetaxel concentration in blood and ascites after drop infusion into each vessel and intraperitoneal cavity of gastric cancer. Jpn J Chemother. 2004;31:2031-4 (Japanese).

16. Yoshida K, Kodera Y, Kochi M, et al. Addition of docetaxel to oral fluoropyrimidine improves efficacy in patients with stage III gastric cancer: interim analysis of JACCRO GC-07, a randomized controlled trial. J Clin Oncol. 2019;37:1296-304.

17. Kodera Y, Ito S, Mochizuki Y, et al. Long-term follow up of patients who were positive for peritoneal lavage cytology: final report from the CCOG0301 study. Gastric Cancer. 2012;15:335-7.

18. Kurokawa Y, Sugimoto N, Miwa H, et al. Phase II study of trastuzumab in combination with S-1 plus cisplatin in HER2-positive gastric cancer (HERBIS-1). Br J Cancer. 2014;110:1163-8.

19. Endo S, Kurokawa Y, Gamoh M, et al. Trastuzumab with S-1 plus cisplatin in HER2-positive advanced gastric cancer without measurable lesions: OGSG 1202. Anticancer Res. 2019;39:1059-65.

20. Kawakami H, Takeno A, Endo S, et al. Randomized, open-label phase II study comparing capecitabine-cisplatin every 3 weeks with S-1-cisplatin every 5 weeks in chemotherapy-naïve patients with HER2-negative advanced gastric cancer: OGSG1105, HERBIS-4A trial. Oncologist. 2018;23:1411-e147147.

21. Sakai D, Satoh T, Kurokawa Y, et al. A phase II trial of trastuzumab combined with irinotecan in patients with advanced HER2positive chemo-refractory gastric cancer: Osaka Gastrointestinal Cancer Chemotherapy Study Group OGSG1203 (HERBIS-5). Jpn J Clin Oncol. 2013;43:838-40.

22. Kawakami H, Fujitani K, Matsuyama J, et al. Comparison of S-1cisplatin every 5 weeks with capecitabine-cisplatin every 3 weeks for HER2-negative gastric cancer (recurrent after S-1 adjuvant therapy or chemotherapy-naïve advanced): pooled analysis of HERBIS-2 (OGSG 1103) and HERBIS-4A (OGSG 1105) trials. Int J Clin Oncol. 2020. https://doi.org/10.1007/s10147-020-01711 $-\mathrm{z}$.

Publisher's Note Springer Nature remains neutral with regard to jurisdictional claims in published maps and institutional affiliations. 